\title{
Method for Controlling the Intensity Distribution of Laser Diode Array Using Computer-generated Hologram
}

\author{
Takayuki Yokoyamaa,", Yosuke Koga ${ }^{\mathrm{a}}$, Yuki Misaki ${ }^{\mathrm{a}}$, Shiyuan Yang ${ }^{\mathrm{a}}$ \\ ${ }^{a}$ Kyushu Institute of Technology, 1-1 Sensui-cho Tobata-ku Kitakyushu-shi Fukuoka-ken, 804-8550, Japan \\ *Corresponding Author: k108126t@ mail.kyutech.jp
}

\begin{abstract}
In recent years, technologies related to laser has been advances, processing technology using the laser has been used in a variety of situations. In this case, the lasers diodes is superior to other lasers in luminous efficacy. In addition, shaping the wavefront of the lasers is ideal in the laser processing. In this paper, we describe the generation method of Kinoform for controlling the intensity distribution of the laser diode. Kinoform is a phase type Computer-generated hologram that does not modulate the amplitude of the light, the utilization efficiency of the light can be improved. Finally, this study can be expected to obtain the intensity necessary for laser processing by controlling using a Kinoform optical intensity distribution of the laser diode array.
\end{abstract}

Keywords: Kinoform, Computer-generated hologram, Fourier transform, Laser diode array.

\section{Introduction}

In recent years, the technology of the laser has advanced. And the processing technique of applying the laser is used in various situations. In particular, we can obtain a high power that is required for the laser processing by using a gas laser or a solid-state laser. However, the luminous efficacy of these lasers is lower than laser diode. Therefore, we propose the use of a laser diode.

The disadvantage of the laser diode is that the output is lower than the output of a gas laser or a solid-state laser. On the other hand, the advantage of the laser diodes is that a high luminous efficacy. Moreover, we can obtain a high output by using a lot of laser diodes because the laser diode is small size and can be integrated.

Generally, the laser has a Gaussian distribution as the intensity of the light becomes maximum at the center and is weak at the peripheral portion. Beam shaping of output light is necessary to increase the quality of application. As the method of this beam shaping, there is a method of using a Computer-generated hologram.

The holograms are recorded amplitude and phase information of the light wave. First, we divided into two laser beams in the optical holography. Second, we irradiated one of the light on the object. And we record the interference pattern of the scattered object beam and the other light in the light-sensitive material. To calculate the diffraction pattern in the computer is a computer-generated holography.

Computer-generated hologram is obtained by recording information that has been calculated by a computer generated holography. Unlike an optics hologram, we can reconstruct the arbitrary intensity distribution of light because recording of Computer-generated hologram doesn't need the model in reality. But recording both the amplitude and phase information calculated by the computer generated holography in the recording medium is difficult. So Computer-generated hologram is recorded one of the information either amplitude or phase. Computer-generated hologram can be classified into two types of the amplitude and phase Computer-generated hologram depending on the recorded information. Amplitude Computer-generated hologram is recorded only the amplitude information. It is easy to create a hologram. However, the utilization efficiency of the light is low. Phase Computer-generated hologram is recorded only the phase information. The reconstruction image error increases because the phase Computer-generated hologram does not have the amplitude information. On the other hand, the utilization efficiency of the light is high since the light is not absorbed in principle. We use the Kinoform which is a phase Computer-generated hologram as an optical device for shaping the beam because the utilization efficiency of the light is higher than amplitude 
Computer-generated hologram.

We use the laser diode array in which a lot of laser diodes arranged in a grid. We think that the coherence between the each diode laser is small because the light interference does not occur unless the light emitted at the same time from the same light source.

\section{Computer-generated hologram}

\subsection{Fourier transform type Computer-generated Hologram}

Figure 1 shows Fourier transform type hologram in the case of the single coherent light. The reconstruction image is projected by screen that left the focus distance through a focus distance remote lens from Kinoform. In this case, the light in the reconstruction image area and light in the Kinoform area are relationship of the Fourier transform pair by function of lens.

Information of Kinoform is obtained by inverse Fourier transformation of the reconstruction image. In addition, information of the reconstruction image is obtained by Fourier transformation of Kinoform. Computer-generated hologram is optimized by this principle.

\subsection{Fourier iterative algorithm}

Gerchberg-Saxton algorithm is known as one of the Fourier iterative algorithm repeats the Fourier transform and inverse Fourier transform. First, the distribution with complex amplitude is Fourier-transformed. Second, it is transposed other amplitude distribution which satisfy certain constraints, and the distribution is inverse Fouriertransformed. Finally, it is transposed amplitude distribution which was input first, and this algorithm return to the first calculation again. Thus this algorithm optimize the intensity distribution of the reconstruction image which is similar to the intensity distribution of the input image.

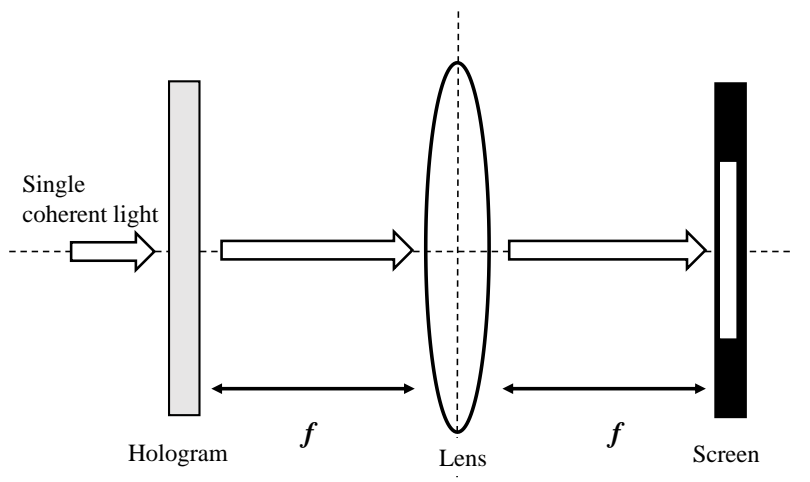

Fig. 1. Fourier transform type hologram.

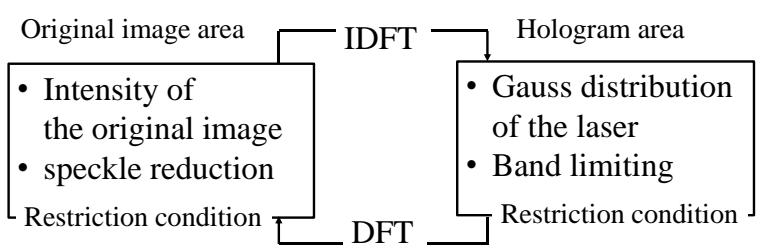

Fig. 2. Fourier iterative algorithm.

\subsection{Phase type Computer-generated Hologram}

When recording the hologram, it is desirable that only one of the information of the amplitude or phase. In this case, the hologram only the phase information is recorded is called phase type hologram. In particular, the phase type computergenerated hologram is called Kinoform.

\subsection{Iterative dummy area method}

The reconstruction image errors occur because it is necessary to a constant amplitude distribution in the Kinoform. Iterative dummy area method has been proposed to reduce the error and promote the convergence of the solution. This method can utilize the amplitude and phase freedom of the dummy area by addition of the dummy area that initial value is zero around the original image. In addition, we limit the band in the area of the hologram as restraint condition in iterative dummy area method. Concretely, we set the value of the amplitude and phase to zero at out-of-band area in hologram side. This condition prevent aliasing error by satisfy the sampling theorem in computer-generated hologram.

\section{Speckle reduction method}

\subsection{Speckle}

Noise called speckle occurs when a hologram is reconstructed in the reconstruction image due to the use of coherent light. Figure 3 is reconstruction image which doesn't use speckle reduction method when Computergenerated hologram is generated. We define phase difference of adjacent four points at the reconstruction image as Fig.4 and formula (1)-(4).

$$
\begin{aligned}
& \Delta \varphi_{0}(x, y)=\varphi(x-1, y-1)-\varphi(x, y-1) \\
& \Delta \varphi_{1}(x, y)=\varphi(x, y-1)-\varphi(x, y) \\
& \Delta \varphi_{2}(x, y)=\varphi(x, y)-\varphi(x-1, y) \\
& \Delta \varphi_{3}(x, y)=\varphi(x-1, y)-\varphi(x-1, y-1)
\end{aligned}
$$




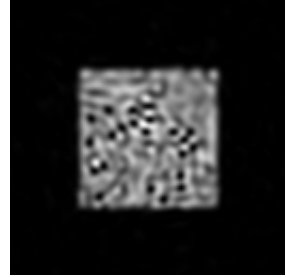

Fig. 3. Speckle in reconstruction image.

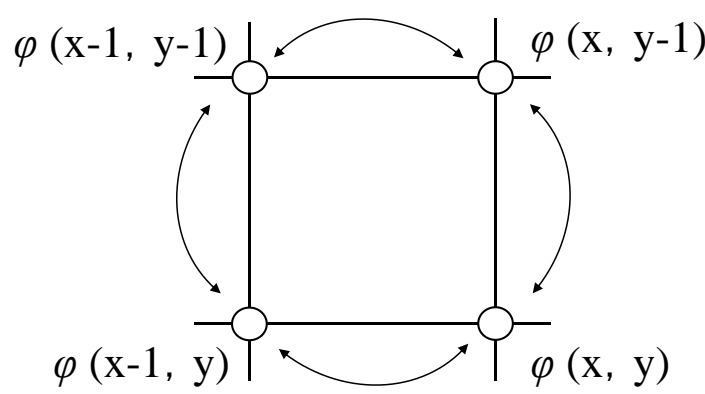

Fig. 4. Phase difference of adjacent four points.

Then, the range become $(-2 \pi, 2 \pi)$ and the sum of phase difference of adjacent four points become zero. However the sum become four values $0, \pm 2 \pi$ or $-4 \pi$ when we wrapping the rang $[-\pi, \pi)$ from the $(-2 \pi, 2 \pi)$. Speckle occurs when the sum of the phase difference is not zero.

\subsection{Iterative speckle reduction method}

In this method, we give one of four points the phase shift that the sum of four points of phase differences becomes zero. The speckle in the reconstruction image is removed by setting this condition as a restriction condition of the Fourier iterative algorithm.

\section{Gibbs phenomenon suppression}

The reconstruction image which has been subjected to band-limited is uneven by the effect of discontinuous points of the intensity distribution of the original image. In order to suppress this phenomenon, we set the original image added a blur to reduce discontinuities as the input signal. In this study, we reduce the high frequency components of the original image used a Gaussian filter. Also, we compare it by changing the kernel size of the filter.

\section{Controlling the intensity distribution of laser diode array}

Using the principle that we showed by the above, we

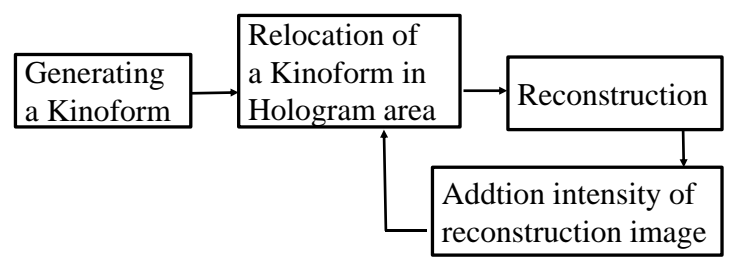

Fig. 5. Flow chart of the calculation.

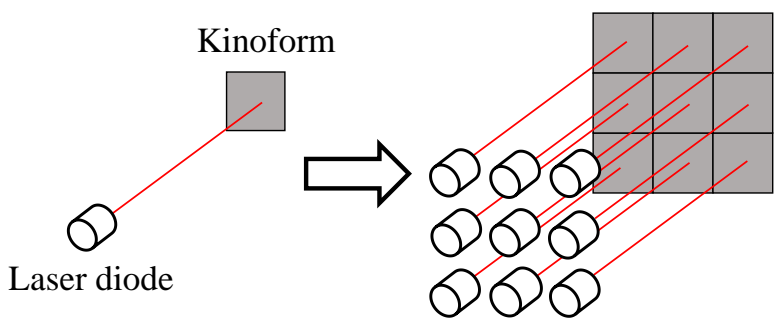

Fig. 6. Relocation of a Kinoform.

generate a Kinoform. We reproduce this Kinoform as many the number of lasers and locates it so that pixels of each Kinoform are not piled up. Then, we make a central coordinate of the optical axis of each laser source of the laser diode array and each Kinoform agree. As shown Fig.6, we locate Kinoforms reproduced so that surround the Kinoform which we located in the center of the dummy area and reconstruct it. The reconstruction is Fourier transform of phase distribution of each Kinoform and the intensity distribution of a supporting laser. If the intensity and phase distribution is the same even if coordinates of the point of the Kinoform are different, the intensity distribution of the reconstruction image does not change by the shift rule. The shift rule shown by formula (5) is one of the properties of the Fourier transform. We can understand that a change in the exponent only by formula (5), but we define $\mathcal{F}[\mathrm{f}(\mathrm{x})]=\mathrm{F}(\mathrm{k})$. In other words, we can't recognize only changing of the phase distribution in the reconstruction image.

$$
\mathcal{F}[f(x-a)]=e^{-i k a} F(k)
$$

Also, we know coherence of the laser each other is small. Therefore, we add the intensity distribution in the reconstruction image from each Kinoform. In this way, we can control the intensity distribution of the reconstruction image which we expect the intensity distribution of light of the laser diode array by Kinoform.

\section{Simulation and results}

We simulate under the following conditions.

- Image size is $64 \times 64$ pixels

- The light source number is 9 
- Dummy area size is 4 times from original image size

- Band limiting is $1 / 2$

We input intensity distribution such as Fig.7 (a) as an original image. In addition, we prepare some images which shaded off an original image by a Gaussian filter. Figure 7 (b) is the image which used a filter to the original image at kernel size as $3 \times 3$ pixels. Figure 7 (c) is the image which used a filter to the original image at kernel size as $5 \times 5$ pixels. We perform the comparison of the output image with these three kinds of input images.

The output images obtained from these input images are Fig.8 (a), Fig.8 (b) and Fig.8 (c). In Fig.8 (a), the speckle reduction is accomplished, and the black spot is gone. However, Gibbs phenomenon appears in Fig.8 (a) by the influence of a discontinuous points of the intensity of light included in Fig.7 (a) which is an input image. We think about the output images which we shade off input images, and decrease a high-frequency component to decrease this Gibbs phenomenon. Figure 8 (b) is the output image which we inputted the image as Fig.7 (b). The reconstruction image becomes brighter generally than Fig.8 (a), and Gibbs phenomenon decreased. However, we can recognize the irregularity in the reconstruction image. Therefore we think

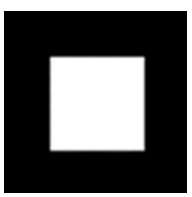

(a)

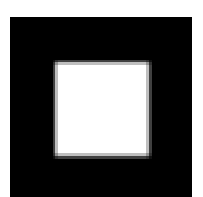

(b)

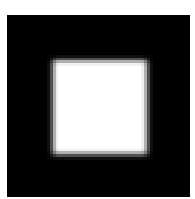

(c)
Fig. 7. Input images.

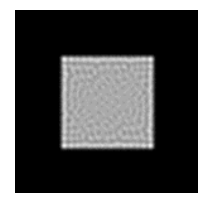

(a)

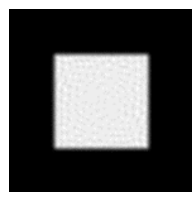

(b)

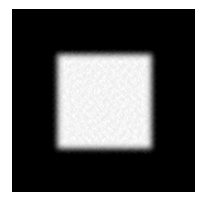

(c)
Fig. 8. Reconstruction images (1000 times iteration).

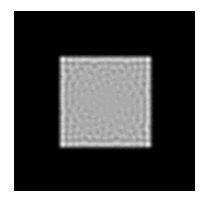

(a)

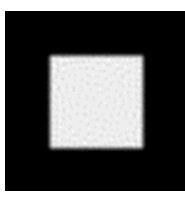

(b)

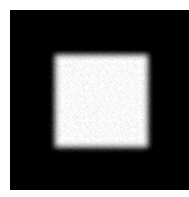

(c)
Fig. 9. Reconstruction images (5000 times iteration). about Fig.8 (c) which is the output image which input Fig.7 (c) which we more shade off. The edge is dimmer than the original image in Fig.8 (c), but the output image more decrease Gibbs phenomenon than Fig.8 (a).

We set the calculation number of times of the Fourier iteration method to 1,000 times in Fig.8 (a), Fig.8 (b) and Fig. 8 (c). In addition, we set the calculation number of times to 5,000 times Fig.9 (a), Fig.9 (b) and Fig.9 (c). We could recognize improvement of the reconstruction image when we observed these.

\section{Conclusion}

In this study, we showed that it is possible to control the intensity distribution of light of laser diode array. As a result, the reconstruction image became better by increase the repetition number of times of Fourier iterative algorithm. In addition, the extreme irregularity by Gibbs phenomenon in the reconstruction image was reduced by shading off an input image by a Gaussian filter moderately.

\section{References}

(1) Toshinori Hora and Shiyuan Yang : "Iterative Dummy Area Method with Flexible Dummy Area Size for the Design of Kinoform" Journal of the Institute of Industrial Applications Engineers, Vol. 1, No. 2, 69-72, 2013

(2) R. W. Gerchberg and W. O. Saxton, Optik 35, 37, 1972

(3) Shiyuan Yang and Hiroaki Takajo "Speckle Reduction of Kinoform Reconstruction Utilizing the $2 \pi$ Ambiguity of Image Phase Differences" Optical Review, Vol. 12, No. 2, 93-96, 2005 